\title{
Sistema de recomendación de música basado en aprendizaje semi-supervisado
}

\author{
J. Roberto Alvarado-García, Janet V. Hernández-García, Esaú Villatoro-Tello, \\ Gabriela Ramírez-de-la-Rosa y Christian Sánchez-Sánchez \\ Departamento de Tecnologías de la Información, \\ División de Ciencias de la Comunicación y Diseño, \\ Universidad Autónoma Metropolitana Unidad Cuajimalpa, México D.F. \\ $\{2113066338,2113066463\}$ @alumnos.cua.uam.mx \\ \{evillatoro, gramirez, csanchez\}@correo.cua.uam.mx
}

\begin{abstract}
Resumen. Actualmente los sistemas de recomendación son cada vez más utilizados por usuario y empresas que buscan por más y mejores contenidos digitales en Internet. Idealmente, los sistemas de recomendación deben aprender los gustos y preferencias de sus usuarios con la intención de facilitarles el proceso de búsqueda. En este trabajo presentamos un método de recomendación musical que es capaz de aprender y de adaptarse a los gustos de sus usuarios sin la necesidad de tener información previa del perfil del usuario. Para la realización de nuestros experimentos utilizamos un subconjunto de datos extraído de la base de datos musical Gracenote. Los resultados obtenidos muestran que con un conjunto reducido de características es posible construir de forma efectiva un modelo de recomendación. Agregado a esto, se muestra que con pocos datos etiquetados es posible obtener resultados aceptables en el problema de recomendación de música.
\end{abstract}

Palabras clave: sistemas de recomendación, recomendación de música, selección de atributos, aprendizaje semi-supervisado, aprendizaje automático.

\section{Introducción}

A medida que el Internet se ha vuelto una fuente importante de información, tanto para usuarios expertos como para usuarios inexpertos, también se ha convertido en un canal importante para la distribución de contenidos digitales muy diversos, por ejemplo: música, vídeo, imágenes, etc. En particular, la búsqueda de música representa una tarea tediosa y a menudo difícil para los usuarios. Una de las razones por las que esto sucede es debido a que los usuarios buscan por contenidos que satisfagan sus gustos personales, razón principal por la cual los sistemas de recomendación se vuelven aplicaciones indispensables 8 .

Las ventajas de un sistema de recomendación no solo aplican al usuario que las utiliza; las empresas dedicadas a la venta de música por Internet pueden obtener atractivas ventajas de este tipo de sistemas. Así por ejemplo, un sistema 
de recomendación puede ser utilizado para ofrecer mayor diversidad de productos (i.e., contenidos musicales) relacionados a los gustos de sus consumidores y en consecuencia incrementar el número de sus ventas. En este sentido, el entender las necesidades del usuario se vuelve un factor fundamental para lograr dichos objetivos 9. Actualmente, este esquema de negocio es seguido por empresas como Netflix 1 , donde el proporcionar a sus clientes contenidos apropiados a sus preferencias les ha permitido volverse un ejemplo exitoso de la utilidad de los sistemas de recomendación que aprenden a partir de los gustos de los usuarios.

Un sistema de recomendación de música (Music Recommendation System o MRS por sus siglas en Inglés) es un sistema con la capacidad de proveer a sus usuarios suscritos recomendaciones musicales tomando en cuenta los gustos y preferencias del usuario o grupo de usuarios [32. Entre los enfoques normalmente empleados por los sistemas de recomendación podemos mencionar seis principales 9, los cuales son: i) Sistemas Basados en Contenido, los cuales se apoyan en la valoración dada por el usuario; ii) Filtrado Colaborativo, basado en las valoraciones que ha realizado una población y realizando un consenso de las mismas; iii) Filtrado Demográfico, basado en las características del usuario como su sexo, país, lenguaje o edad; iv) Basados en Comunidad, utilizada en las redes sociales se apoya en la valoración de las amistades del usuario para realizar recomendaciones; v) Basados en Conocimiento, son sistemas que utilizan la información proporcionada por el usuario para otorgar una clasificación, y vi) Filtrado Híbrido, que combina dos o más de los tipos de sistemas de recomendación mencionados. En la sección 2 se dan más detalles de este tipo de enfoques.

En este trabajo se propone una herramienta para la recomendación de contenidos musicales basada en atributos de contenido. El sistema propuesto aprende los gustos musicales del usuario conforme éste lo utiliza al aplicar técnicas de aprendizaje de máquind2 ${ }^{2}$. Para la realización de nuestros experimentos utilizamos instancias obtenidas de la base de datos Gracenot 3 Los resultados obtenidos muestran que: 1) con una pequeña cantidad de atributos es posible hacer recomendaciones pertinentes a un conjunto de usuarios, y 2) el método propuesto converge hacia un desempeño aceptable después de pocas interacciones con el mismo.

El resto del documento se encuentra organizado de la siguiente manera, en la sección 2 se describen algunos de los enfoques previamente utilizados así como ejemplos de algunos sistemas de recomendación actualmente activos en Internet. En la sección 3 se describe la configuración del método propuesto. La sección 4 se describen los datos empleados para la realización de este trabajo, así como los objetivos y resultados obtenidos de nuestros experimentos. Finalmente en la sección 5 se mencionan nuestras principales conclusiones y algunas ideas de trabajo futuro.

\footnotetext{
${ }^{1}$ Empresa que distribuye contenidos visuales (https://www.netflix.com)

2 También conocido como Aprendizaje Automático por algunos autores [7].

3 http://www .gracenote.com
} 
Sistema de recomendación de música basado en aprendizaje semi-supervisado

\section{Trabajo relacionado}

El objetivo general de esta sección es proveer al lector de los antecedentes necesarios para conocer el funcionamiento de los sistemas de recomendación de música. En primer lugar se comentan las técnicas comúnmente empleadas para proponer sistemas de recomendación, y en segundo lugar se de da una breve descripción de los sistemas de recomendación de música que actualmente se encuentran activos en Internet.

\subsection{Métodos de recomendación}

Como se mencionó en la sección anterior, según [9] existen seis esquemas distintos empleados en los sistemas de recomendación. Sin embargo, en el problema de recomendación musical se habla de dos enfoques principales, i.e., sistemas basados en contenido y sistemas basados en filtrado colaborativo.

En los sistemas basados en contenido, los datos (i.e., canciones) que han sido vistos y calificados en algún momento previo, son empleados para construir/enriquecer un perfil de usuario; en otras palabras, tratan de aprender los gustos del usuario a partir del contenido que él ha visitado. Estos seudo-perfiles son empleados posteriormente para encontrar y recomendar más contenidos. Para lograrlo, el esquema tradicional busca aquellas canciones con una similitud alta con aquellos contenidos que el usuario ha visto antes. Ejemplos de este tipo de sistemas son los descritos en [13]. Un elemento clave en este tipo de sistemas es la adecuada representación de los contenidos así como la existencia y veracidad de un perfil del usuario, ambos aspectos impactarán de manera directa en la efectividad de las recomendaciones hechas por el sistema.

Como alternativa al cálculo de similitudes entre los contenidos musicales y los perfiles de usuario, los sistemas basados en filtrado colaborativo miden similitudes entre perfiles de usuarios. La idea intuitiva de este tipo de técnicas es que usuarios con gustos similares pueden ser objeto de recomendaciones similares. De esta forma, usuarios con perfiles similares son vistos como un grupo (i.e., un meta-perfil) de usuarios que comparten ciertas características. El objetivo principal del filtrado colaborativo es entonces proporcionar de forma masiva recomendaciones a usuarios con el mismo meta-perfil. Ejemplos de este tipo de sistemas son 210. Bajo el enfoque de filtrado colaborativo, los sistemas dependen de la efectiva construcción de los meta-perfiles de usuarios además de que son propensos a hacer recomendaciones poco efectivas debido a la diversidad que puede ser producto de la naturaleza de los grupos de usuarios considerados.

\subsection{Aplicaciones de recomendación}

Entre los sistemas de recomendación de música más conocidos a nivel mundial esta Pandora 4 Pandora nace de un proyecto llamado Music Genome Project, el

\footnotetext{
4 http://www.pandora.com/
} 
cual clasifica la música en base a su melodía, armonía, ritmo, instrumentación, orquestación, arreglos, letra y otros elementos, en total utilizan 450 características. Estas características son analizadas para realizar recomendaciones completamente personalizadas a cada usuario suscrito en la plataforma. La gran ventaja de Pandora es su enorme base de conocimientos mediante la cual obtiene detalles muy específicos de las canciones que contiene. Como principales desventajas están la disponibilidad restringida a unos cuantos países y el costo que implica usarla.

Una aplicación similar a Pandora es Last.fm[5 un servicio de código abierto fundado en 2002. Last.fm es una red social, una radio vía Internet y además un sistema de recomendación de música que construye perfiles y estadísticas sobre gustos musicales, basándose en los datos enviados por los usuarios registrados. Usa un esquema de filtrado colaborativo para sugerir listados de reproducción a usuarios con el mismo meta-perfil. Entre las ventajas de Last.fm están el uso de etiquetas referidas al género, humor o características del artista que permiten al usuario escuchar canciones con etiquetas que le podrían interesar. Otra ventaja es su complemento Audioscrobbler que puede construir un perfil de usuario con la biblioteca de música de su computadora personal. Por último Last.fm crea sus listas de acuerdo al número de reproducciones de una canción y no al número de ventas, ofreciendo una estadística más real de la música escuchada. Como desventajas encontramos que al igual que Pandora, Last.fm ofrece suscripciones bajo el pago de una cuota. Además, el esquema de filtrado colaborativo no ofrece una recomendación a nivel de usuario sino a nivel comunidad.

Otro ejemplo de aplicación es Moodagent 6 Moodagent, a diferencia de Last.fm y Pandora, emplea como parte de sus características para representar las canciones a las emociones contenidas en las mismas. Esto en combinación con atributos perceptuales (atributos extraídos de la señal acústica) permiten a Moodagent hacer recomendaciones a sus usuarios. Una de las principales desventajas de Moodagent es que requiere de un gran conjunto de datos etiquetados para identificar adecuadamente las preferencias de sus usuarios, agregado a esto, Moodagent no implementa ninguna estrategia que le permita ajustarse al perfil de usuario conforme éste utiliza la aplicación.

El método propuesto en este trabajo retoma algunas de las ideas de los sistemas de recomendación basados en contenido. La diferencia principal del método propuesto es que éste no requiere de tener información del perfil de usuario para proponer recomendaciones. En su lugar proponemos una arquitectura que permite a un algoritmo de aprendizaje automático ajustarse a los gustos y preferencias del usuario conforme éste va utilizando la aplicación. La ventaja principal del esquema propuesto es la posibilidad de tener recomendaciones $100 \%$ personalizadas.

5 http://www.lastfm.es

6 http://www.moodagent.com 


\section{Método propuesto}

La figura 1 muestra de forma esquemática la arquitectura del sistema propuesto. Nótese que un elemento importante dentro del sistema es el usuario, pues es él quien a través del Módulo de Retroalimentación permitirá a los algoritmos de aprendizaje conocer sus gustos y preferencias musicales. Es importante mencionar que bajo este esquema, la primera vez que el usuario utiliza la aplicación del MRS, ésta proporcionará una selección aleatoria (y lo más diversificada posible) de contenidos musicales al usuario. El Módulo de Retroalimentación estará encargado de identificar aquellos contenidos que el usuario haya marcado como favoritos. Idealmente la retroalimentación que el usuario proporciona al MRS no debe ser hecha de forma explícita. El sistema podría configurarse para detectar cuándo el usuario escucha contenidos completos una o varias veces y de esta forma proporcionar una retroalimentación de forma indirecta. Así entonces, los objetos identificados como favoritos serán enviados al conjunto de datos etiquetados, y posteriormente tras haber pasado por el proceso de Extracción de Atributos apoyar a la Construcción del Clasificador. El resultado de estos procesos será un modelo más adecuado a las preferencias musicales del usuario. Posteriormente dicho modelo es empleado para clasificar y recomendar nuevo contenido al usuario, el cual previo a mostrarse, atraviesa por un proceso de Ranking, el cual permite identificar aquellas recomendaciones en las que se tiene mayor confianza respecto a su clasificación. Así entonces, se espera que el MRS converja a un modelo adecuado a los gustos y preferencias del usuario conforme éste utilice el sistema.

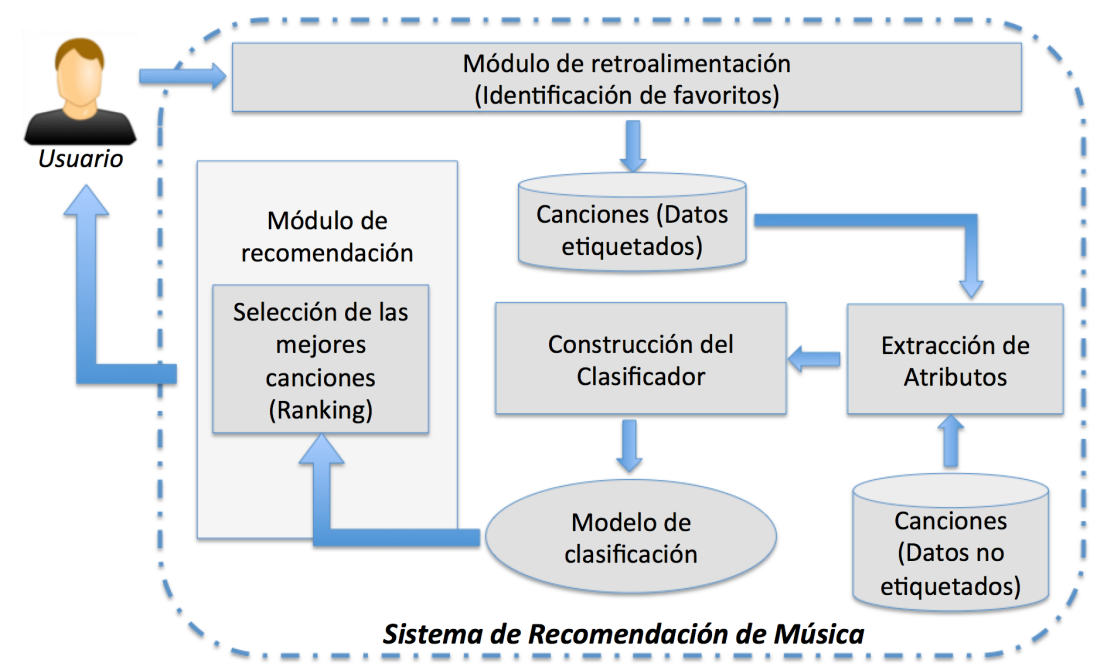

Fig. 1. Arquitectura del modelo de clasificación $100 \%$ personalizado y re-entrenado conforme el usuario provee retroalimentación al sistema. 
En resumen, abordamos el problema de recomendación de contenidos musicales como un problema de clasificación binaria, donde a cada canción se le asigna la etiqueta "recomendable" o "no-recomendable". El sistema propuesto aplica técnicas de aprendizaje semi-supervisado para ir afinando y mejorando el modelo de clasificación conforme más datos etiquetados se van obteniendo. El aprendizaje semi-supervisado aprovecha la información que es posible obtener tanto de los datos no etiquetados como de los pocos datos etiquetados disponibles. Es importante mencionar que en el aprendizaje semi-supervisado ambos conjuntos de datos (i.e., no-etiquetados y etiquetados) deben provenir del mismo dominio, además de que son técnicas recomendadas cuando los datos etiquetados son escasos [11.

\subsection{Conjunto de Datos}

Debido a la falta de un corpus adecuado a nuestros propósitos, nos dimos a la tarea de la construcción y etiquetado de un conjunto de datos que nos permitirá evaluar la pertinencia de nuestro método propuesto. Para esto obtuvimos datos del sitio en línea Gracenote, una filial de Tribune Media Company, empresa encargada de distribución de contenido por medios digitales.

Gracenote cuenta actualmente con más de 100 millones de canciones, las cuales están etiquetadas con gran diversidad de meta-datos. En el nivel más alto de organización se identifican seis grandes categorías de estos meta-datos, los cuales son: i) género de la canción, ii) humor, iii) año de grabación, iv) velocidad de la canción, v) origen o región más asociada al artista y vi) sexo del cantante. De entre estas categorías seleccionamos ocho sub-tipos de atributos para la representación de los datos, los cuales se describen en la tabla 1 .

Tabla 1. Tabla que describe los atributos seleccionados para la representación de los contenidos musicales contenidos en la base de datos de Gracenote.

\begin{tabular}{|c|c|c|}
\hline Nombre & Valores & Descripción \\
\hline ID canción & $\mathrm{N} / \mathrm{A}$ & Identificador de canción \\
\hline Título & $\mathrm{N} / \mathrm{A}$ & Título de la canción \\
\hline Autor & $\mathrm{N} / \mathrm{A}$ & Autor de la canción \\
\hline Año de lanzamiento & 8 generaciones & $\begin{array}{l}\text { Periodo de tiempo al que pertenece } \\
\text { la canción }\end{array}$ \\
\hline Genero & 59 géneros & $\begin{array}{l}\text { Divide la música en diferentes esti- } \\
\text { los de acuerdo a sus letras, instru- } \\
\text { mentos y ritmo }\end{array}$ \\
\hline Lenguaje & 11 idiomas y 1 sin letra & Ubica el lenguaje de las canciones \\
\hline Tempo & 7 velocidades & $\begin{array}{l}\text { El tempo equivale a la velocidad de } \\
\text { la canción }\end{array}$ \\
\hline Estado de ánimo & 25 emociones & $\begin{array}{l}\text { Los tipos de emoción que tiene una } \\
\text { canción }\end{array}$ \\
\hline
\end{tabular}


Es importante mencionar que los primeros tres atributos, aunque se utilizan en la interfaz gráfica de nuestro sistema, no son empleados por el proceso de aprendizaje al momento de construir el modelo de clasificación. Agregado a esto, los cinco atributos restantes, i.e., año de lanzamiento, género, lenguaje, tempo y estado de ánimo, fueron utilizados como atributos nominales en el proceso de entrenamiento y clasificación de las instancias.

El conjunto total de datos empleados en la fase experimental fue de 120 canciones, de las cuales 60 fueron marcadas como positivas (recomendables) y 60 como negativas (no-recomendables). Para el proceso de etiquetado de los datos se tomaron en cuenta las valoraciones, hechas a mano, de dos usuarios con perfiles similares.

\subsection{Algoritmos de aprendizaje}

Dado que nuestra propuesta para identificar contenidos musicales recomendables para un usuario no depende de ningún algoritmo de aprendizaje en particular, podemos emplear prácticamente cualquier clasificador para enfrentar el problema. Para los experimentos realizados seleccionamos dos diferentes algoritmos de aprendizaje, los cuales son algoritmos representativos dentro de la gran variedad de algoritmos de aprendizaje disponibles actualmente en el campo de aprendizaje computacional [46]. Específicamente, consideramos los siguientes:

- Naïve Bayes(NB). Método probabilístico que asume la independencia de los atributos entre las diferentes clases del conjunto de entrenamiento.

- J48. Un algoritmo que permite generar un árbol de decisión, el cual selecciona los atributos más discriminativos basándose en su medida de entropía.

En nuestros experimentos se empleó la implementación de Weka $[5]$ de cada uno de estos algoritmos empleando los parámetros por defecto. Es importante mencionar que para todos los experimentos se aplicó como estrategia de validación la técnica de validación cruzada a diez pliegues.

\subsection{Evaluación}

Para evaluar el método propuesto se utilizaron las medidas tradicionales para evaluación de sistemas de clasificación, tales como precisión, recuerdo y medida $F$ [7]. La precisión $(P)$ es la proporción de instancias clasificadas correctamente en una clase $c_{i}$ con respecto a la cantidad de instancias clasificadas en esa misma clase. El recuerdo $(R)$, la proporción de instancias clasificadas correctamente en una clase $c_{i}$ con respecto a la cantidad de instancias que realmente pertenecen a esa clase. Así, la precisión se puede ver como una medida de la corrección del sistema, mientras que el recuerdo da una medida de cobertura o completitud.

Normalmente se emplea la medida $F$ para describir el comportamiento de la clasificación, la cual se define como: 


$$
F=\frac{\left(1+\beta^{2}\right) P * R}{\beta^{2}(P+R)}
$$

donde $\beta$ representa la media armónica entre la precisión y el recuerdo. La función de $\beta$ es la de controlar la importancia relativa entre las medidas de precisión y recuerdo. Es común asignar un valor de 1 indicando igual importancia a ambas medidas.

\section{Experimentos y resultados}

Como se ha mencionado en secciones anteriores, el objetivo principal de este trabajo fue determinar hasta qué punto es posible construir un sistema de recomendación de música, siguiendo un enfoque tradicional de clasificación automática, capaz de determinar cuándo una canción es probablemente atractiva para un usuario en particular. Para lograr dicho objetivo se propusieron dos conjuntos de experimentos, mismos que se describen a continuación:

Experimento 1. Evaluar el impacto que tiene emplear los atributos seleccionados como forma de representación de las canciones, i.e., atributos nominales, en el proceso de recomendación de música. En este experimento se plantea un esquema completamente supervisado. La hipótesis principal de este experimento sugiere que con pocos atributos y muchos datos etiquetados es posible construir un modelo que permita identificar de manera eficiente los gustos de un usuario.

Experimento 2. Evaluar el comportamiento del sistema propuesto en un escenario lo más real posible, es decir, considerando la retroalimentación proporcionada por el usuario. En otras palabras, se simula un esquema de aprendizaje semi-supervisado. La hipótesis principal de este experimento sugiere que tras pocas iteraciones, el sistema de recomendación puede lograr un desempeño comparable al obtenido en el Experimento 1.

Los resultados del Experimento 1 se muestran en la tabla 2 y tabla 3 . Como se mencionó antes, se evaluó el desempeño de dos algoritmos de clasificación (J48 y Naïve Bayes) en la tarea de identificar canciones recomendables para un usuario en particular. Los resultados se reportan en términos de precisión $(P)$, recuerdo $(R)$ y la medida $F$ (F-measure). Agregado a esto, se evaluó la pertinencia de cada uno de los atributos seleccionados de forma individual. Así entonces, cada fila de las tablas 2 y 3 representa el desempeño obtenido por el clasificador respectivo utilizando sólo el atributo correspondiente. La última fila de ambas tablas (i.e., Todos) se refiere al desempeño del clasificados empleando los cinco atributos como forma de representación de las canciones.

Los resultados mostrados en la tabla 2 reflejan el desempeño obtenido por los algoritmos de clasificación en el problema de clasificación binaria, es decir, identificando la clase "recomendable" y "no-recomendable". Como es posible observar, ambos métodos de clasificación, i.e., J48 y Naïve Bayes obtienen un desempeño 
Tabla 2. Resultados obtenidos en la tarea de recomendación de música empleando un esquema de aprendizaje supervisado. Para todos los experimentos se empleó una estrategia de validación cruzada de diez pliegues.

\begin{tabular}{rcccc|ccc}
\hline \multirow{2}{*}{$\begin{array}{l}\text { Atributos } \\
\text { empleados }\end{array}$} & \multicolumn{3}{c}{ Algoritmos de clasificación/Medidas de Evaluación } \\
\cline { 2 - 4 } \cline { 6 - 8 } & \multicolumn{3}{c}{$\mathbf{J 4 8}$} & & \multicolumn{3}{c}{ Naïve Bayes } \\
\cline { 2 - 3 } \cline { 6 - 8 } & $P$ & $R$ & $F$ & & $P$ & $R$ & $F$ \\
\hline Genero & 0.884 & 0.875 & 0.874 & & $\mathbf{0 . 8 8 4}$ & $\mathbf{0 . 8 7 5}$ & $\mathbf{0 . 8 7 4}$ \\
Año & 0.659 & 0.658 & 0.658 & & 0.659 & 0.658 & 0.658 \\
Lenguaje & 0.636 & 0.608 & 0.587 & & 0.636 & 0.608 & 0.587 \\
Edo. ánimo & 0.742 & 0.742 & 0.742 & & 0.742 & 0.742 & 0.742 \\
Tempo & 0.617 & 0.617 & 0.617 & & 0.617 & 0.617 & 0.617 \\
\hline Todos & $\mathbf{0 . 8 8 4}$ & $\mathbf{0 . 8 7 5}$ & $\mathbf{0 . 8 7 4}$ & 0.859 & 0.858 & 0.858 \\
\hline
\end{tabular}

comparable. Esto indica, hasta cierto punto, que los atributos empleados son apropiados para la tarea de clasificación abordada y que los resultados no dependen del algoritmo de clasificación.

Nótese que emplear sólo el atributo de Género para entrenar los algoritmos de clasificación permite obtener muy buenos resultados de clasificación. Es igualmente valioso hacer notar que el resto de los atributos, de forma independiente, aportan información valiosa al clasificador, logrando valores de $F$ que varían de 0.62 a 0.73 . En general, de estos experimentos podemos concluir que los árboles de decisión (J48) obtienen los mejores resultados de clasificación, y al mismo tiempo, también podemos decir que solo el atributo de género es el más importante en el proceso de clasificación.

Tabla 3. Resultados obtenidos identificando sólo a los elementos de la clase positiva (i.e., música "recomendada"). Para todos los experimentos se aplicó una técnica de validación cruzada de diez pliegues.

\begin{tabular}{|c|c|c|c|c|c|c|}
\hline \multirow{3}{*}{$\begin{array}{l}\text { Atributos } \\
\text { empleados }\end{array}$} & \multicolumn{6}{|c|}{ Algoritmos de clasificación/Medidas de Evaluación } \\
\hline & \multicolumn{3}{|c|}{$\mathrm{J} 48$} & \multicolumn{3}{|c|}{ Naïve Bayes } \\
\hline & $P$ & $R$ & $F$ & $P$ & $R$ & $F$ \\
\hline Genero & 0.941 & 0.800 & 0.865 & 0.941 & 0.800 & 0.865 \\
\hline$A \tilde{n} o$ & 0.667 & 0.633 & 0.650 & 0.667 & 0.633 & 0.650 \\
\hline Lenguaje & 0.575 & 0.833 & 0.680 & 0.575 & 0.833 & 0.680 \\
\hline Edo. ánimo & 0.746 & 0.733 & 0.739 & 0.746 & 0.733 & 0.739 \\
\hline Tempo & 0.609 & 0.650 & 0.629 & 0.609 & 0.650 & 0.629 \\
\hline Todos & 0.941 & 0.800 & 0.865 & 0.841 & 0.883 & 0.862 \\
\hline
\end{tabular}


Los resultados de la tabla 3 muestran el desempeño de los algoritmos empleados al momento de identificar solo los elementos de la clase positiva, es decir, clasificando las instancias "recomendables". El objetivo de esta tabla de resultados es mostrar qué configuración es capaz de obtener el mejor desempeño en la identificación de las canciones recomendables. La razón de hacer esto se debe a que en un escenario real, un usuario preferirá tener pocas recomendaciones pero muy precisas; en otras palabras, un sistema que tenga una baja presencia de falsos positivos. Así entonces, el método que permite tener menor tasa de falsos positivos son los árboles de decisión, pues logran superar en 10 puntos porcentuales al método de Naïve Bayes.

Con la intención de identificar la pertinencia de cada uno de los atributos empleados durante los experimentos se hizo un análisis de ganancia de información. Los resultados de este análisis se muestran en la tabla 4. Del análisis de ganancia de información se puede concluir que todos los atributos tienen información valiosa. Debido a que no hay atributos que cumplan $I G \leq 0$, concluimos que todos los atributos son pertinentes en el problema de clasificación abordado y en consecuencia es preferible conservarlos.

Tabla 4. Análisis de Ganancia de Información sobre los cinco atributos empleados para representar las canciones de nuestra base de datos.

\begin{tabular}{rc}
\hline Atributo & $\mathbf{I G}$ \\
\hline Genero & 0.8275 \\
Edo. ánimo & 0.5131 \\
Año & 0.1841 \\
Lenguaje & 0.1259 \\
Tempo & 0.0657 \\
\hline
\end{tabular}

Finalmente, la figura 2 muestra los resultados obtenidos del Experimento 2. De acuerdo a la descripción que se dio de la arquitectura del sistema en la sección 3 (figura 1), el usuario juega un papel importante debido a la retroalimentación (directa o indirecta) que puede proporcionar al sistema respecto de sus gustos. Bajo esta configuración, el sistema propuesto deberá ser capaz de entrenar un modelo de clasificación para recomendación de música conforme el usuario utiliza la aplicación. Como se mencionó antes, esta configuración se asemeja a un esquema de aprendizaje semi-supervisado, es decir, se tienen pocos elementos etiquetados al principio y conforme se va utilizando el clasificador, i.e., después de varias iteraciones, se incrementa la colección de datos etiquetados con los cuales el modelo de clasificación puede ser re-entrenado con la finalidad de adecuarse más al concepto que se quiere aprender, en este caso los gustos musicales del usuario.

En la figura 2 se simuló la retroalimentación que podría proporcionar un usuario al momento de interactuar con el sistema. Así entonces, en el eje horizontal se indica la cantidad de ejemplos etiquetados con los que se entrena y 


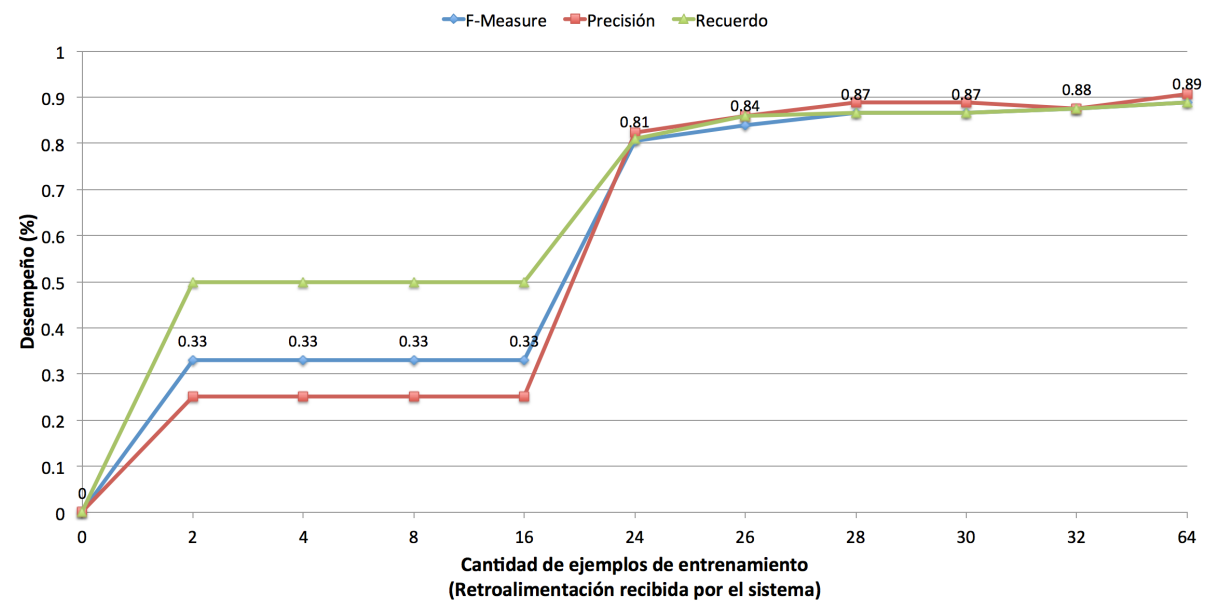

Fig. 2. Resultados simulando el proceso de retroalimentación.

genera el modelo de recomendación (i.e., 2, 4, 8, 16, 24, ...). Los resultados se reportan en términos de $P, R$ y $F$-measure. Es importante mencionar que en la figura 2 se reporta el promedio de haber hecho estos experimentos empleando cinco diferentes muestreos de los datos, esto como forma de validación de los experimentos realizados.

Los resultados obtenidos de este experimento muestran que con 2 ejemplos etiquetados el sistema logra alcanzar un desempeño de $F=0.33$, y tan solo después de haber proporcionado retroalimentación sobre 24 ejemplos, el sistema de recomendación logra obtener ya un $F=0.81$. Es importante hacer notar que a partir de este punto el modelo de recomendación tiende a estabilizarse, alcanzando en una $F=0.89$ con 64 instancias etiquetadas.

En conclusión, estos experimentos reafirman la pertinencia del método propuesto, y muestran cómo el enfoque semi-supervisado es capaz de obtener resultados comparables contra un enfoque completamente supervisado.

\section{Conclusiones y trabajo futuro}

En este trabajo hemos presentado un método de recomendación de música que aprende y se adapta a los gustos del usuario. El método propuesto se abordó como un problema de clasificación binaria y por tanto emplea técnicas de aprendizaje automático para la construcción del modelo de recomendación.

Entre los objetivos que se plantearon al inicio del trabajo fue por un lado, evaluar la pertinencia de una serie de atributos que son fácilmente extraíbles de la base de datos musicales Gracenote. Y por otro lado, evaluar la efectividad del método bajo un enfoque semi-supervisado, es decir, con muy pocos datos etiquetados. 
Los resultados obtenidos mostraron que los atributos proporcionados por la base de Gracenote son apropiados para el problema abordado. Fue interesante observar que bajo un esquema $100 \%$ supervisado, el atributo Genero proporciona información muy valiosa al momento de construir el modelo de recomendación. Sin embargo una serie de experimentos adicionales, mostraron que todos los atributos empleados poseen valores altos de ganancia de información, razón por la cual se decidió conservarlos en los experimentos posteriores. Agregado a esto, dado que ambos algoritmos de aprendizaje empleados son capaces de enfrentar la ausencia de un atributos (i.e., datos faltantes), si en un determinado momento una canción extraída de la base de datos carece del atributo Genero, el modelo será capaz de asignar una categoría si cuenta con algo de información en el resto de los atributos.

Por otro lado, un segundo bloque de experimentos mostraron que con pocos datos etiquetados es posible construir un modelo de recomendación adaptado a los gustos del usuario. Bajo un enfoque semi-supervisado se pudo observar que con apenas 2 datos etiquetados el método logra una $F=0.33$, y con solo 24 datos el método alcanza un desempeño comparable al obtenido cuando se emplea todo el conjunto de datos etiquetados disponible (i.e., $F=0.81$ ). Estos resultados son alentadores, pues indican que el método propuesto es apropiado para identificar "rápidamente" los gustos de los usuarios y además de que puede adaptarse a los mismos conforme el usuario va proporcionando retroalimentación.

Como trabajo futuro inmediato se propone incluir alguna serie de atributos perceptuales, es decir, atributos extraídos de la señal acústica. Ejemplos de este tipo de atributos son la densidad del pitch, atributos asociados a la frecuencia y amplitud de la señal acústica, valores de entropía, etc. Nuestra intuición es que dichos atributos pueden enriquecer favorablemente el modelo de recomendación propuesto.

Agradecimientos. Agradecemos al Departamento de Tecnologías de la Información de la Universidad Autonoma Metrópolitana Unidad Cuajimalpa y al proyecto CONACYT número CB2010/153315 por el apoyo otorgado para la realización de este trabajo.

\section{Referencias}

1. Basu, C., Hirsh, H., Cohen, W.: Recommendation as classification: Using social and content-based information in recommendation. In: Proceedings of the Fifteenth National Conference on Artificial Intelligence. pp. 714-720. AAAI Press (1998)

2. Bu, J., Tan, S., Chen, C., Wang, C., Wu, H., Zhang, L., He, X.: Music recommendation by unified hypergraph: Combining social media information and music content. In: Proceedings of the International Conference on Multimedia. pp. 391-400. MM '10, ACM, New York, NY, USA (2010), http://doi.acm.org/10.1145/1873951. 1874005

3. Chen, H.C., Chen, A.L.P.: A music recommendation system based on music data grouping and user interests. In: Proceedings of the Tenth International Conference 
on Information and Knowledge Management. pp. 231-238. CIKM '01, ACM, New York, NY, USA (2001), http://doi.acm.org/10.1145/502585.502625

4. Duda, R., Hart, P.: Pattern classification and scene analysis. Wiley (1996), http: //www.ica.luz.ve/ enava/redesn/ebooks/DHS/Versi\%F3nPS/DHSChap4.ps

5. Garner, S.R.: Weka: The waikato environment for knowledge analysis. In: Proc. of the New Zealand Computer Science Research Students Conference. pp. 57-64 (1995)

6. Hastie, T., Tibshirani, R., Friedman, J.: The Elements of Statistical Learning: Data Mining, Inference, and Prediction. Springer (2009)

7. Mitchell, T.: Machine Learning. McGraw-Hill (1997)

8. Park, H.S., Yoo, J.O., B., C.S.: A context-aware music recomendation system using fuzzy bayesian networks with utility theory. Fuzzy Systems and Knowledge Discovery 4223, 970-979 (2006)

9. Ricci, F., Rokach, L., Shapira, B., Kantor, P.B.: Recommender Systems Handbook. Springer (2011)

10. Su, J.H., Yeh, H.H., Yu, P.S., Tseng, V.S.: Music recommendation using content and context information mining. IEEE Intelligent Systems 25(1), 16-26 (Jan 2010), http://dx.doi.org/10.1109/MIS.2010.23

11. Zhu, X., Goldberg, A.B.: Introduction to Semi-Spuervised Learning. Morgan and Claypool (2009) 